To the Editors:

\title{
Re: Ingestion of dug well water from an area with high prevalence of chronic kidney disease of unknown aetiology (CKDu) and development of kidney and liver lesions in rats - Toxicological viewpoint
}

\section{T B Ananda Jayalal ${ }^{1}$}

Ceylon Medical Journal 2018; 63: 97-98

DOI: http://doi.org/10.4038/cmj.v63i2.8678

\section{Introduction}

Development of kidney and liver lesions in rats following long term (15 months) ingestion of water from three dug wells was described by Thammitiyagodage et al in the $C M J$. Water from three wells selected from CKDu prevalent villages (assumed to contain toxins) were given separately to three test groups of rats and tap water from Colombo given to the fourth (control) group. It should be noted that CKDu cases were reported from only two of the selected villages (Medirigiriya and Bisobandaragama) but not from the third (Divuldamana/Dibulagala), which is at direct variance from the experimental framework. It had been assumed but not proven that Colombo tap water was free of potential toxins.

Further it had been assumed that either the rats' diet was free of any potential toxin, or if present, since all rats were exposed to the same diet, such toxic effect can be disregarded. This design of study methodology grossly violates principles of toxicology; viz (i) the dose effect relationship, and (ii) the total exposure from all possible routes (water, food, air, skin etc.) of any potential toxin needs to be considered in order to arrive at credible conclusions. As such, the design of this toxicological study is deficient in the context of current environmental toxicology and CKD knowledge.

Also, CKDu and CKD are used somewhat casually in the paper and without apparent rigour. Coincidental causes of CKD like hyperglycaemia have not been considered, nor have the rats been sampled for any such pre-existing CKD precursors or markers (for example, the authors have presumed absence of zero glomerular/ interstitial lesions in the rats prior to commencement of the study. Such shortcomings can be confusing to the reviewer and will only serve to dilute the conclusion.

If the objective of the study to identify the causative factors or associative factors of $\mathrm{CKDu}$, researchers might have also tested kidney and liver injury bio markers in humans who consume the same (experimental) water. If a similar association was found, it could have triggered a deeper analysis using animal or other suitable models.

It should be noted that while a large number of parameters can be measured in assessing water quality, the article provides analysis of only five elements. Testing for pesticide residues was notably absent - this could have been useful in interpreting the results of the study. Lead and mercury are also potential candidate toxins which were excluded. For reference, WHO water quality guidelines provide a comprehensive list of possible contaminants.

The authors have ignored potential food contaminants in the study. Food and water intake by the rats have also not been quantified. Data relating to food contaminants and quantities of food and water ingested if available would have allowed the estimation of various ingested contaminants.

Apparently test rats were found to sustain acute kidney and liver injury vs. their control counterparts. However, corresponding injuries to humans who consume the same have not been noted. Instead CKD is reported in two wells but not the third (Divuldamana/Dimbulagala), which is presumed to be safe for humans. In contrast, the authors suggest that small animals tend to eliminate

Post Grad Certificate in Paed. Nutrition, Director, Youth, Elderly and Disabled Persons National Consultant Biosafety Policy Development Project, FAO, Environmental Toxicologist, Food Contaminant Specialist, Ministry of Health, Sri Lanka.

Correspondence: TBAJ, e-mail: <jayalal313@yahoo.co.uk>. Received 18 February 2018 and revised version accepted 22 February 2018. 
metabolites more rapidly than humans. This suggests that the outcomes detected in the study have potential to be more marked in humans.

It is mentioned that the main lesions found consistently among the experimental animals were "partial to complete sclerosis of glomeruli" and "glomerular basement membrane thickening", which are histological features more consistent with diabetes mellitus and hypertension in humans rather than reported in $\mathrm{CKDu}$. There is however, reference to interstitial nephritis in rats from all three groups, although occurring in fewer cases than showed glomerular sclerosis. This suggests that the rats may have had diabetes mellitus or hypertension (or precursors thereof) prior to the study, thereby pointing to a flawed screening / selection process.

A consistent theme of hepatocellular carcinoma, hepatitis and adenoma formation is reported. Are the authors alluding to the detected toxic agents also having a carcinogenic effect, and if so, how does this fit in with epidemiological studies of humans in the three test villages?

The authors further say "No significant difference was observed in serum creatinine levels in test groups ( $p>0.05$ ). No significant difference observed in serum cystatin $C$ levels ( $p>0.05$ ) after 9 months of the experimental period". Such observations indicate that chronic renal impairment does not exist in the subjects, but this is not acknowledged.

Reduction of GFR in test animals is reported. As the quantitative water consumption data is not available, other reasons for low GFR (e.g. low consumption or dehydration from any number of causes) cannot be excluded.

One inference that can be made from the results is that all the rats are exposed to some kind of environmental insult (because the control group also shows some lesions, although this is more pronounced in the test rats). The possibility of infection, presence of immunosuppressive agents or acutely toxic substance that lead to hepato/ renal damage, carcinogen in the test water has not been excluded in the context of current environmental toxicology knowledge in this study. Similarly, a large number of confounders need to be considered in drawing conclusions from the study.

Toxins in fertiliser and water can easily be absorbed and concentrated by food plants; therefore, the dose of such toxins taken up by humans from food is several orders of magnitude greater than from water. As such, this study reiterates the need to understand, in parallel, the food habits of affected families, as well as their exposure to toxins via other potential sources.

Considering all the above facts, the conclusion "that rats who ingested water from high disease prevalent villages were susceptible to interstitial nephritis. Ingestion of fluoride alone or in combination with some other unmeasured component in water may be responsible for renal and hepatic changes in Wistar rats" appears incorrect (at least the phrase "Fluoride alone") do not lead to possible association of fluoride with human $\mathrm{CKD}$ or $\mathrm{CKDu}$; nor is it a credible model to extrapolate to human health effects. Therefore, the results cannot be used to draw reasonable conclusions in order to design an effective public health intervention.

In addition, to test a commodity that humans are exposed to naturally, either wilfully or unintentionally, should not warrant animal studies unless something of experimental value that cannot be measured in humans is to be measured. Hence this study also perhaps unintentionally also violates the ethics of animal welfare.

\section{References}

1. Thammitiyagodage MG1, Gunatillaka MM, Ekanayaka N et al. Ingestion of dug well water from an area with high prevalence of chronic kidney disease of unknown etiology $(\mathrm{CKDu})$ and development of kidney and liver lesions in rats. Ceylon Med J. 2017; 62(1): 20-24. 